\title{
Microsatellite Instability-High, Malignant Insulinoma With Brain Metastasis
}

\author{
Jason Starr $^{1}$, Guillermo Puebla ${ }^{2}$, Jessica McMillan ${ }^{3}$, Jason T. Lewis ${ }^{4}$, Pashtoon M. Kasi ${ }^{5}$ \\ 1. Hematology/Oncology, Mayo Clinic, Jacksonville, USA 2. Hematology/Oncology, University of Puerto Rico, Medical \\ Sciences Campus, San Juan, PRI 3. Clinical Genomics, Mayo Clinic, Jacksonville, USA 4. Department of Laboratory \\ Medicine and Pathology, Mayo Clinic, Jacksonville, USA 5. Medical Oncology, University of Iowa, Iowa City, USA
}

Corresponding author: Jason Starr, starr.jason@mayo.edu

\begin{abstract}
Insulinomas are the most common type of functional pancreatic neuroendocrine tumor. Although insulinomas usually are noninvasive or benign, $10 \%$ are deemed invasive or malignant. The pathologic mechanisms that lead to the malignant phenotype are not well elucidated. In this case report, we present a patient with stage 4 malignant insulinoma with metastasis to the liver, bone, and brain. Genetic analysis of the tumor showed that the tumor was mismatch-repair deficient and had a high rate of microsatellite instability. There was loss of MLH1 - and PMS2-encoded protein expression, and MLH1 and MEN1 variants were identified. Notably, the liver metastasis showed considerable tumor heterogeneity (well differentiated) compared with the brain metastasis (poorly differentiated).
\end{abstract}

Review began $07 / 24 / 2021$ Review ended 08/02/2021 Published 08/07/2021

๑) Copyright 2021 Starr et al. This is an open access article distributed under the terms of the Creative Commons Attribution License CC-BY 4.0., which permits unrestricted use, distribution, and reproduction in any medium, provided the original author and source are credited.
Categories: Endocrinology/Diabetes/Metabolism, Gastroenterology, Oncology

Keywords: pancreatic neuroendocrine tumors, pancreatic insulinoma, msi- high, cancer-immunotherapy, men 1

\section{Introduction}

Insulinomas are pancreatic neuroendocrine tumors (panNETs) that originally were thought to originate from islet cells ( $\beta$ cells), however, more recent studies have suggested that the progenitor cell is from the acinar/ductal system [1]. Although most panNETs are nonfunctional, insulinomas account for approximately $60 \%$ of the functional panNETs [2,3]. Patients can present with the classic Whipple triad, which consists of symptoms of hypoglycemia, documented low blood sugar at the time of symptoms, and reversal of symptoms with glucose administration. Symptoms of hypoglycemia can include confusion, visual changes, and unusual behavior (e.g. staring off into space). Further, hypoglycemia results in the activation of the sympathetic-adrenal axis which can result in diaphoresis, palpitations, and tremulousness. At diagnosis, approximately $90 \%$ of insulinomas are localized and are considered benign [2,4]. PanNETs are classified on the basis of morphologic differentiation and proliferation rate (Ki-67 and mitotic index) [5].

Most panNETs are nonhereditary (sporadic) tumors, but approximately $10 \%$ are attributable to inherited disorders such as multiple endocrine neoplasia type 1 (MEN1 syndrome), von Hippel-Lindau syndrome, neurofibromatosis type 1 , or tuberous sclerosis [6]. MEN1 syndrome is a hereditary condition characterized by development of multiple tumors in hormone-producing glands such as the parathyroid glands, anterior pituitary gland, and pancreas.

Patients with MEN1 syndrome have an $80 \%$ to $100 \%$ chance of panNET development, and $20 \%$ of these panNETs will be an insulinoma [4]. Because this disease is typically localized, the mainstay of treatment is surgical resection. The 10-year risk of recurrence is higher for patients with MEN1 syndrome (20\% for patients with MEN1, 5\% for patients without MEN1). For patients with malignant (ie, metastatic) insulinoma, the 10 -year survival rate is approximately $30 \%$, whereas it is approximately $90 \%$ for patients with localized disease [7].

\section{Case Presentation}

We describe a case of malignant insulinoma that is notable for the following unique aspects: the tumor was mismatch-repair deficient and microsatellite instability-high (dMMR/MSI-high), the patient had MLH1 (somatic) and MEN1 (germline) sequence variants, and a brain metastasis was identified with a gallium 68 (68Ga) DOTATATE positron emission tomography-computed tomography (PET-CT) scan. The reporting of this clinical case is in compliance with the CARE guidelines [8].

A 59-year-old White woman presented to the emergency department with the chief concern of refractory hypoglycemia. A physical examination showed unremarkable findings. Pertinent laboratory test results included a glucose level of $46 \mathrm{mg} / \mathrm{dL}$ (reference range, 70-100); chromogranin A, 1,559ng/mL (reference, <93); insulin, 27.1mcIU/mL (reference, 2.6-24.9); and C-peptide, 6.5ng/mL (reference, 1.1-4.4). A magnetic resonance image of the abdomen with contrast showed a pancreatic tail mass, along with innumerable liver lesions and retroperitoneal lymphadenopathy (Figure 1). 


\section{Cureus}

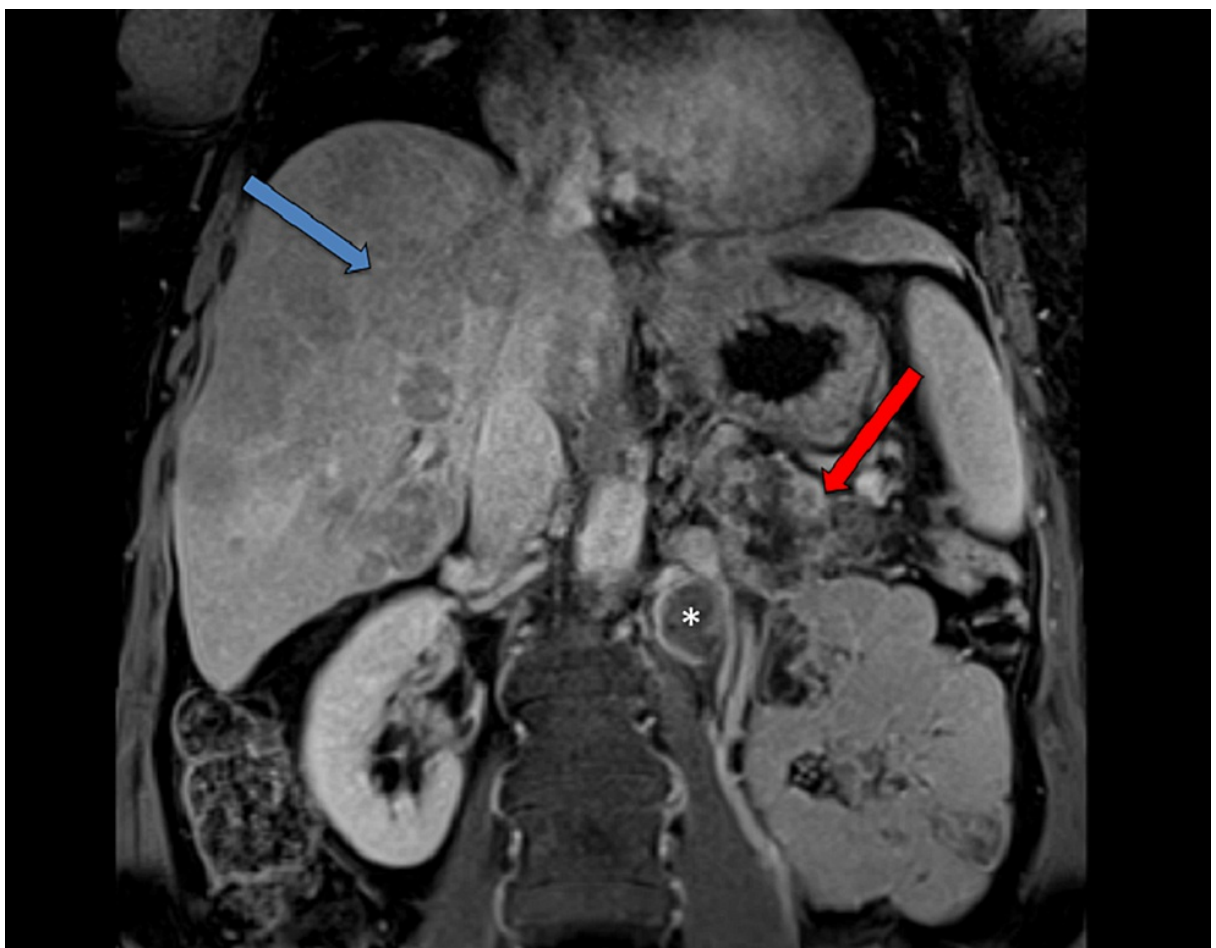

FIGURE 1: Magnetic resonance imaging (coronal) shows a pancreatic tail mass (red arrow), liver metastasis (blue arrow), and retroperitoneal lymphadenopathy (asterisk).

A biopsy specimen, taken from a liver lesion, showed a well-differentiated neuroendocrine tumor with a Ki67 of 30\% (grade 3) (Figures 2, 3).

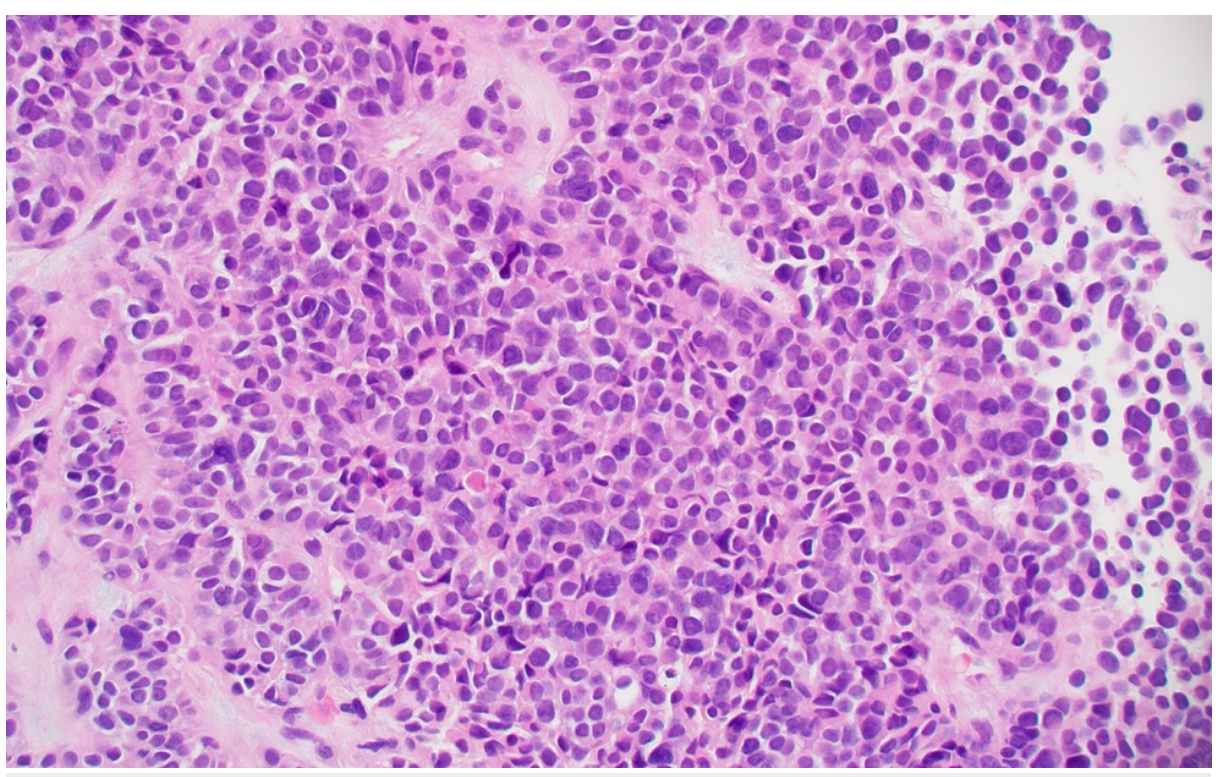

FIGURE 2: Liver, well-differentiated neuroendocrine tumor (hematoxylineosin, original magnification $\times 200$ ). 


\section{Cureus}

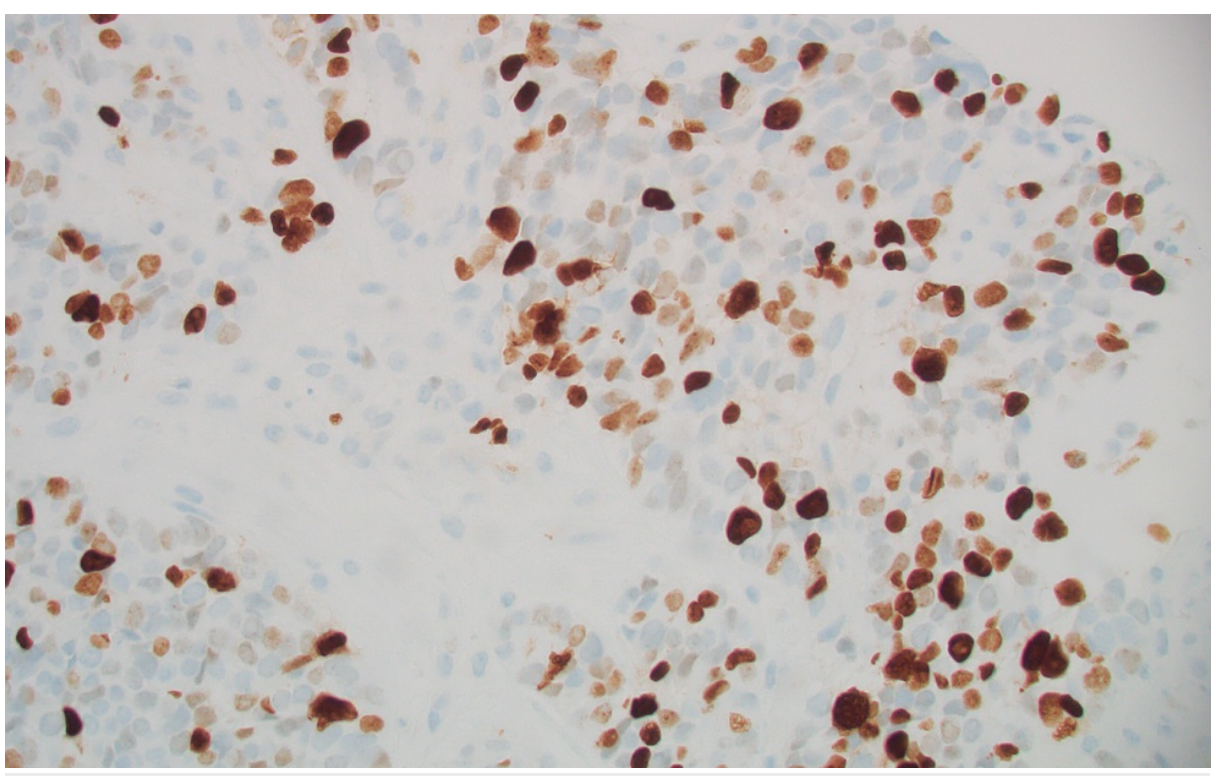

FIGURE 3: Liver, Ki-67 immunohistochemical stain (original magnification $x$ 400). The proliferation rate was estimated to be $30 \%$.

Given the patient's hypoglycemia, she received intravenous infusions of $10 \%$ glucose and octreotide, and diazoxide treatment was initiated. That same day, an interventional radiologist performed a bland embolization to the right lobe of the liver. The patient's blood glucose level subsequently stabilized, and she was discharged.

In the clinic, the patient started treatment with 5-fluorouracil, leucovorin, and oxaliplatin (mFOLFOX6). The patient achieved a partial response, but progressive sensorimotor peripheral neuropathy developed after five months and mFOLFOX6 was discontinued. The patient had one cycle of capecitabine and temozolomide, but she had severe cytopenia and rapid progression of disease in the abdomen. A 68Ga DOTATATE PET-CT scan was obtained and identified a large right parietal brain tumor (approximately $3.3 \mathrm{~cm}$ ) with associated edema, mass effect, and mild midline shift, along with a left frontal lobe lesion (Figure 4). The scan also showed a pancreatic tail lesion, hepatic metastasis, and uptake in the right pelvis (Figure 5). 


\section{Cureus}

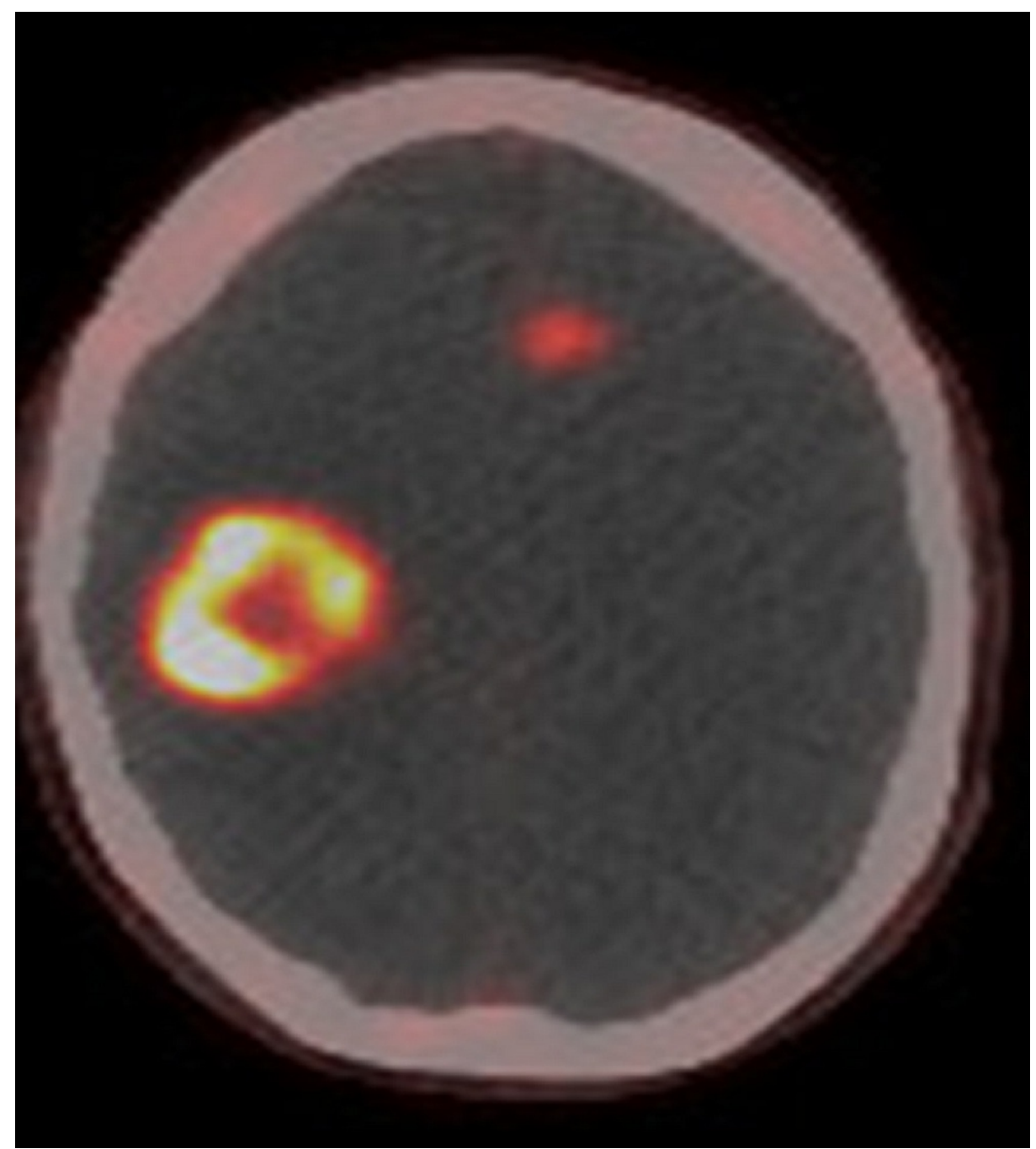

FIGURE 4: 68Gallium DOTATATE positron emission tomography-computed tomography imaging (axial) of the brain shows a right parietal lesion and left frontal lesion. 


\section{Cureus}

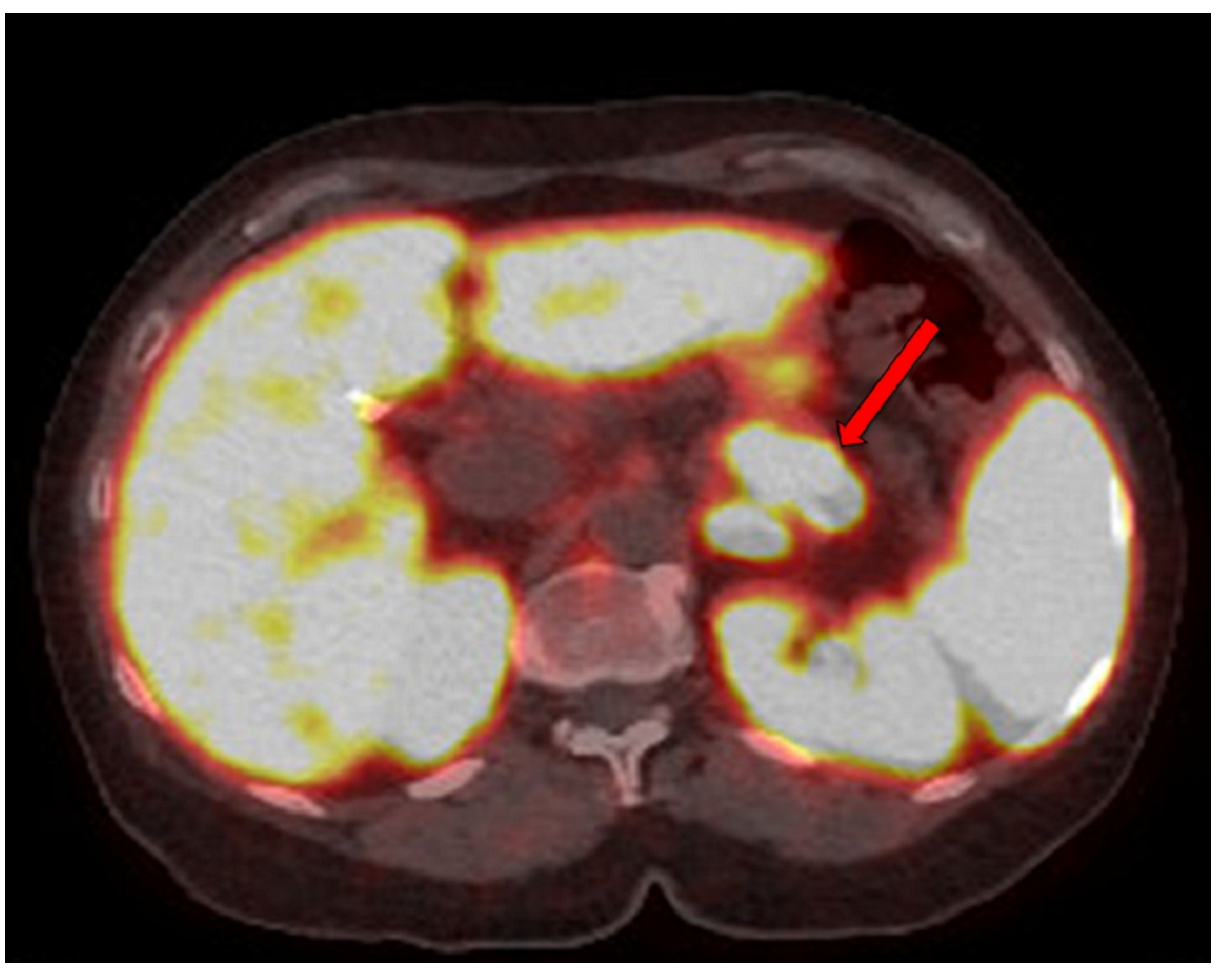

FIGURE 5: 68Gallium DOTATATE positron emission

tomography-computed tomography imaging (axial) shows a pancreatic tail lesion (red arrow).

She underwent a right craniotomy and resection of the metastatic lesion. Pathologic analysis showed a poorly differentiated neuroendocrine carcinoma with a Ki-67 of 90\% (Figures 6, 7).

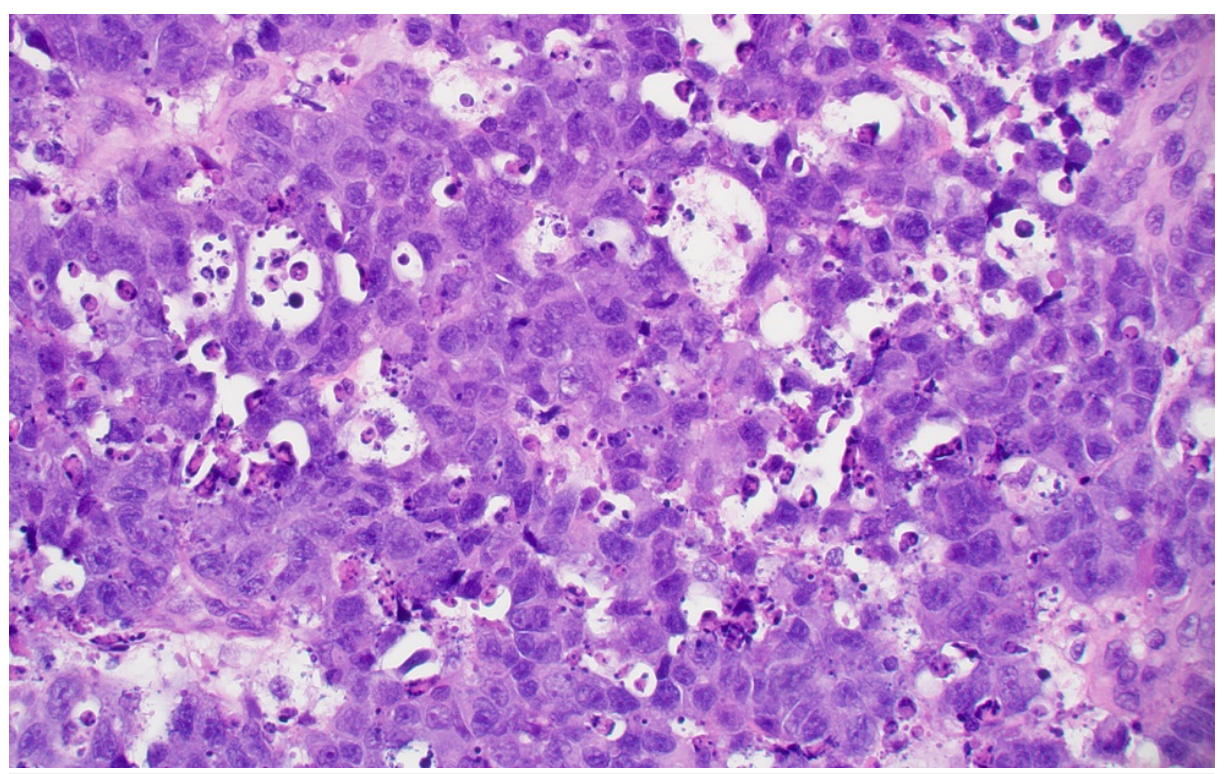

FIGURE 6: Brain, poorly differentiated neuroendocrine tumor

(hematoxylin-eosin, original magnification $x 400$ ). 


\section{Cureus}

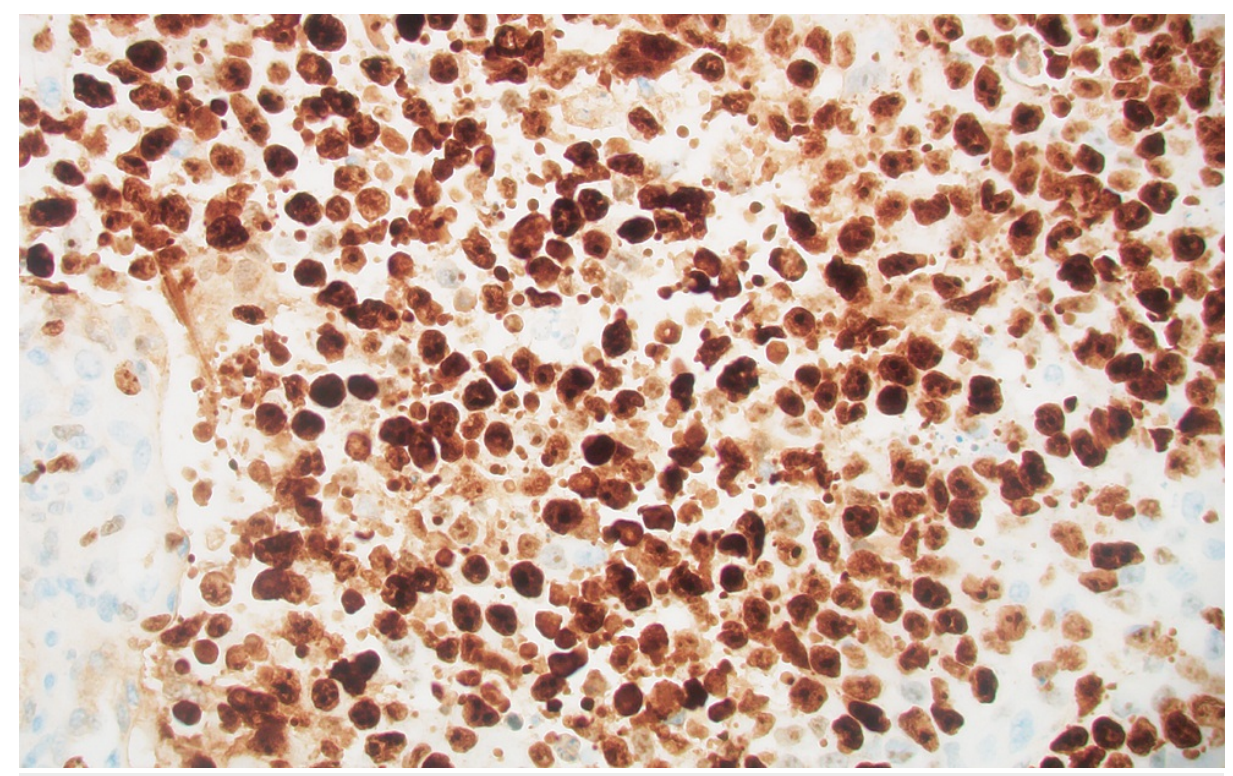

FIGURE 7: Brain, Ki-67 immunohistochemical stain (original magnification $x 400$ ). The proliferation rate was estimated to be $90 \%$.

Next-generation sequencing of the original liver biopsy specimen was performed after the surgery and showed pathologic sequence variations in the MEN1 and MLH1 genes (Table 1).

\begin{tabular}{|c|c|c|c|}
\hline Pathogenic sequence variant & Protein alteration & Exon & DNA alteration \\
\hline MEN1 (germline) & R516fs & 10 & c.1546delC \\
\hline MLH1 (somatic) & K255fs & 9 & c.762 763delGA \\
\hline
\end{tabular}

TABLE 1: Genetic Features of the Malignant Insulinoma

The tumor had an intermediate mutational burden (eight variants/Mb). Immunohistochemical (IHC) analysis of the tumor showed loss of MLH1 protein expression (weakly positive, +1 ) but no PMS2 expression. MSH6 and MSH2 protein expression were normal at 2+ (Figures 8, 9, 10, 11). 


\section{Cureus}

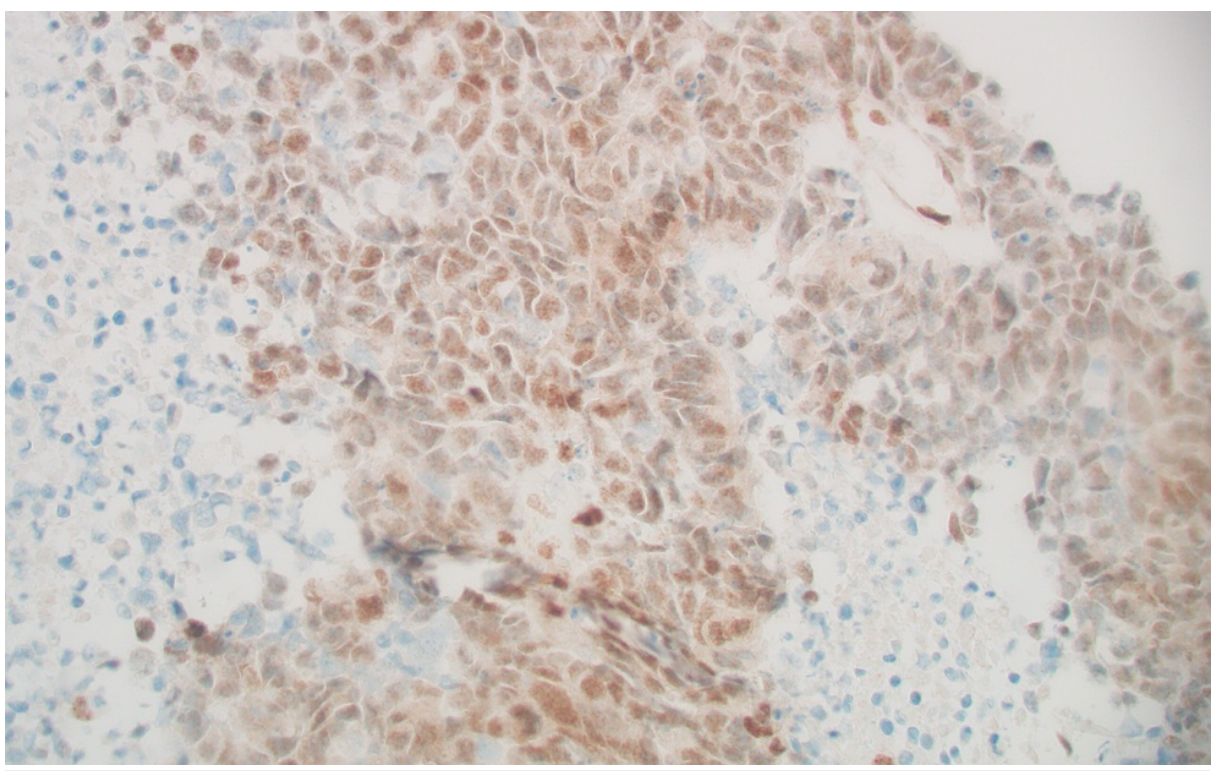

FIGURE 8: MLH1, weakly positive (+1).

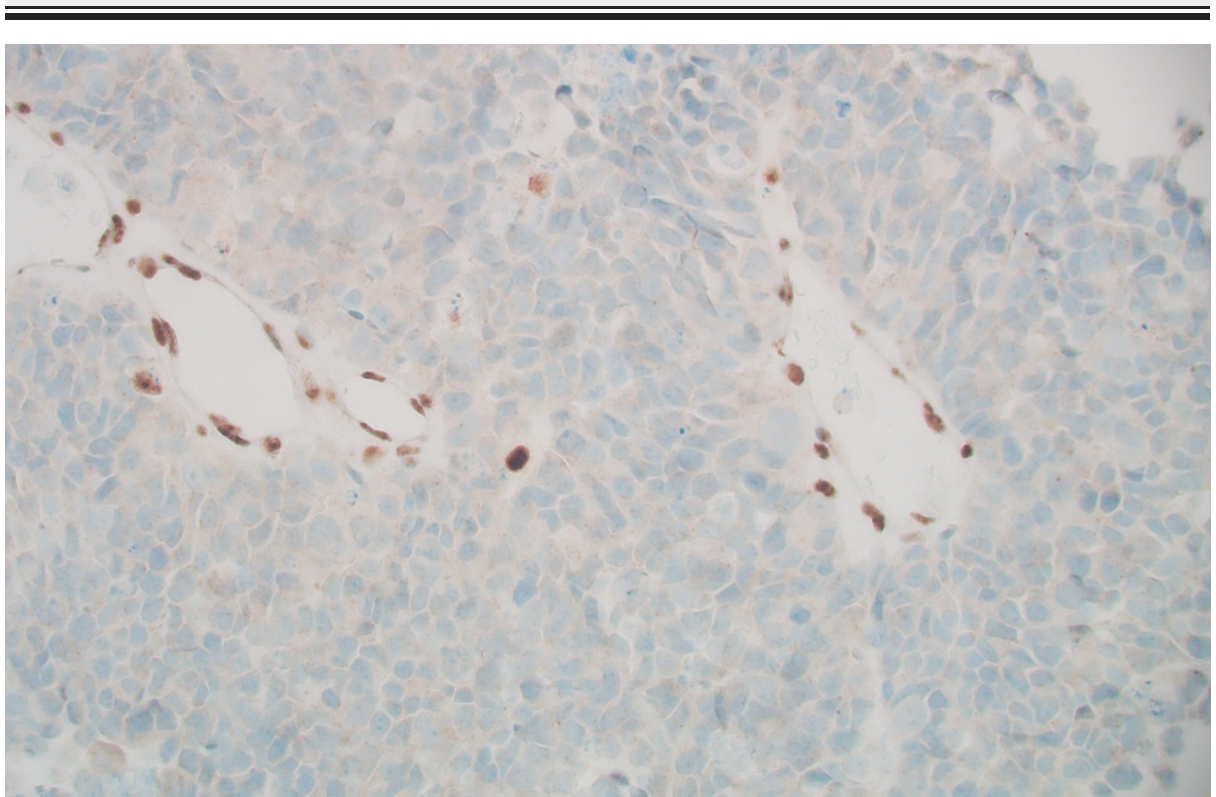

FIGURE 9: PMS2, absent. 


\section{Cureus}

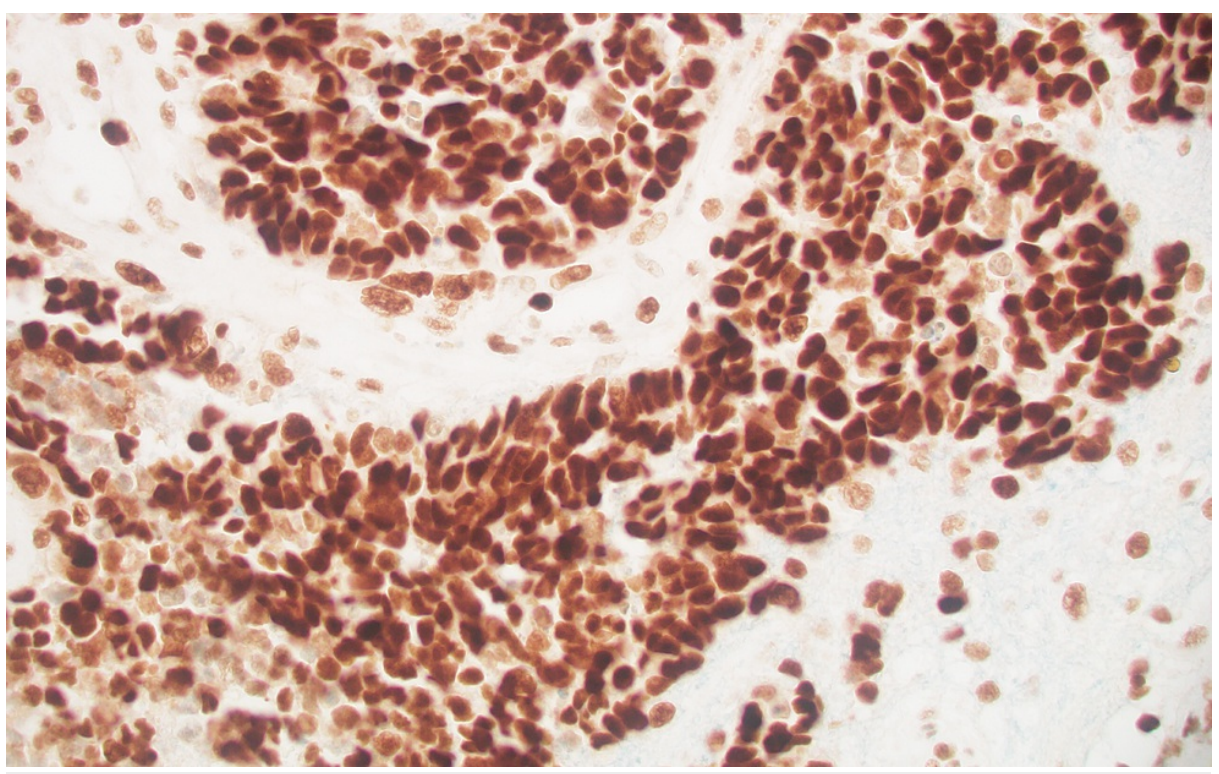

FIGURE 10: MSH2, positive (+2).

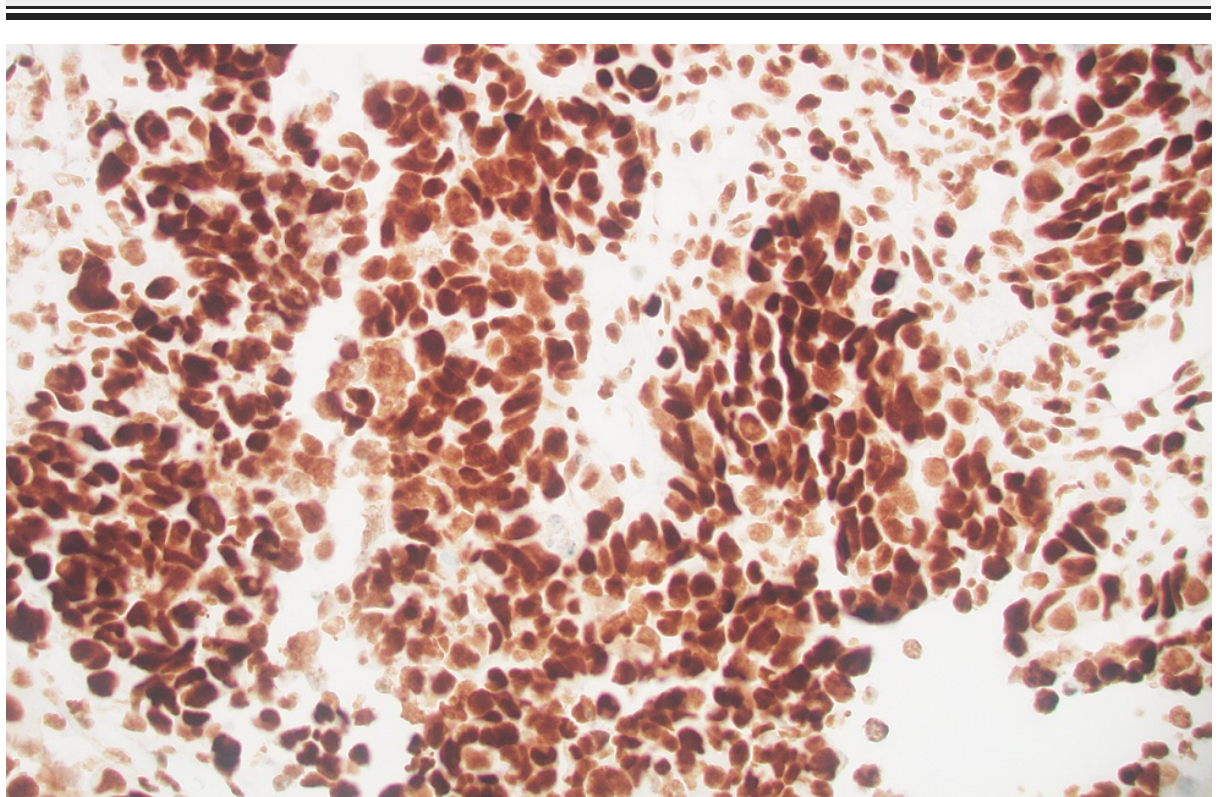

FIGURE 11: MSH6, positive (+2).

Immunostaining with the SP142 antibody showed no PD-L1 expression. Additionally, polymerase chain reaction testing confirmed the MSI-H status. Of note, the brain pathology specimen was not sent for nextgeneration sequencing.

Given the molecular findings of dMMR/MSI-H, the patient promptly started treatment with the anti-PD-1 antibody, pembrolizumab. The patient received two doses, but unfortunately, her clinical status quickly declined because of increasing abdominal pain, progressive weakness, and failure to thrive. The patient entered hospice care and died shortly thereafter.

\section{Discussion}

\section{Genetics of MEN1, MLH1, and MSI-H}

Using multiplex, next-generation sequencing, we identified pathologic frameshift sequence variations in MEN1 (germline variant) and MLH1 (somatic variant) (Table 1). The MEN1 gene, first discovered in 1988, is located on chromosome 11q13 and encodes the protein menin. MEN1 is a tumor-suppressor gene involved in cell cycle regulation, but the exact mechanism of tumorigenesis in MEN1 variants is not completely understood. Somatic variants of MEN1 have been identified in several nonhereditary tumors such as 
parathyroid adenomas, gastrinomas, and insulinomas [9,10]. Loss of heterozygosity of MEN1 can lead to MEN1 syndrome. Notably, 25\% to 44\% of MEN1 variants identified in panNETs are somatic in nature [11-13]. The variant identified in our patient is a known germline disease-associated allele (ClinVar database: https://www.ncbi.nlm.nih.gov/clinvar/variation/200999/). Our patient did not have a known family history of MEN1 syndrome.

MLH1 is part of a gene family (which includes MSH2, MSH3, MSH6, and PMS2) that is responsible for the surveillance and repair of DNA base mismatches (ie, mismatch repair), in which loss leads to dysregulated DNA repair. The human genome has long stretches of conserved repetitive DNA sequences that help protect the coding regions. These stretches are prone to variation, and thus, if mismatch repair is dysregulated, errors can accumulate and ultimately result in MSI-H. This faulty DNA repair is best exemplified by Lynch syndrome, an inherited autosomal dominant disease, in which variants in mismatch repair genes lead to increased risk of various malignancies, most commonly colon and uterine cancer. Studies have shown that up to $36 \%$ of panNET cases have MLH1 loss. One mechanism by which this occurs is hypermethylation of the gene $[14,15]$. Mei et al. studied 55 sporadic insulinomas and showed that 20 (36\%) showed reduced expression of MLH1 (defined as <25\% positive with IHC staining) or loss of MLH1 (determined with IHC), which corresponded to a $33 \%$ rate of MSI-H [16].

\section{Detection of brain metastasis with 68Ga DOTATATE PET-CT}

Brain metastasis of well-differentiated neuroendocrine tumors is rare, affecting an estimated $1.5 \%$ to $5 \%$ of cases [16]. In our review of the literature, we identified only two prior reports of insulinoma with brain metastasis $[17,18]$. The $68 \mathrm{Ga}$ DOTATATE PET-CT scan has dramatically increased the ability to detect previously occult disease [19]. The sensitivity of this imaging technique is shown in our case because it identified intracranial disease (brain metastasis) in a patient who had no neurologic symptoms at the time. It should be noted that meningiomas also have somatostatin expression and thus will exhibit uptake, which could lead to a false-positive result in a patient with neuroendocrine disease.

\section{Conclusions}

This case report describes a patient with a malignant insulinoma with some unique features, including MEN1 and MLH1 sequence variants, dMMR/MSI-H status from loss of MLH1, and identification of brain metastasis with 68Ga DOTATATE PET-CT. It is important to point out the heterogeneity of the tumor, which presented as a well-differentiated neuroendocrine tumor and later manifested with poorly differentiated morphology in the brain. Also of interest was the lack of response to pembrolizumab, even though the tumor was dMMR/MSI-H. Based on the above case and the available literature, we recommend testing patients with panNETs, specifically insulinomas, for the presence or absence of MMR proteins or MSI status.

\section{Additional Information}

\section{Disclosures}

Human subjects: Consent was obtained or waived by all participants in this study. Conflicts of interest: In compliance with the ICMJE uniform disclosure form, all authors declare the following: Payment/services info: All authors have declared that no financial support was received from any organization for the submitted work. Financial relationships: Jason Starr declare(s) personal fees from Pfizer. Jason Starr declare(s) personal fees from Tersera. Jason Starr declare(s) personal fees from Advanced Accelerated Applications. Jason Starr declare(s) personal fees from Ipsen. Other relationships: Pashtoon M. Kasi Advisory/Consultancy: $\bullet$ Taiho Oncology (to institution) $\bullet$ Ipsen (to institution) $\bullet$ Natera $\bullet$ Foundation Medicine $\bullet$ Merck $\bullet$ AstraZeneca $\bullet$ Bayer $\bullet$ Daiiche Sankyo (AZ) $\bullet$ Delcath Pashtoon M. Kasi: Research/Trial Support (to institution) $\bullet$ BMS $\bullet$ Celgene $\bullet$ Astrazeneca $\bullet$ BTG/Boston Scientific $\bullet$ Advanced Accelerator Applications $\bullet$ Array Biopharma $\bullet$ RenovoRx $\bullet$ Amgen $\bullet$ Tersera $\bullet$ Seagen $\bullet$ Roche/Genentech.

\section{References}

1. Vortmeyer AO, Huang S, Lubensky I, Zhuang Z: Non-islet origin of pancreatic islet cell tumors. J Clin Endocrinol Metab. 2004, 89:1934-8. 10.1210/jc.2003-031575

2. Metz DC, Jensen RT: Gastrointestinal neuroendocrine tumors: pancreatic endocrine tumors . Gastroenterology. 2008, 135:1469-92. 10.1053/j.gastro.2008.05.047

3. Falconi M, Plockinger U, Kwekkeboom DJ, et al.: Well-differentiated pancreatic nonfunctioning tumors/carcinoma. Neuroendocrinology. 2006, 84:196-211. 10.1159/000098012

4. Jensen RT, Berna MJ, Bingham DB, Norton JA: Inherited pancreatic endocrine tumor syndromes: advances in molecular pathogenesis, diagnosis, management, and controversies. Cancer. 2008, 113:1807-43. 10.1002/cncr.23648

5. Neoplasms of the neuroendocrine pancreas. WHO Classification of Tumours of Endocrine Organs. Lloyd RV, Osamura RY, Klöppel G, Rosai J (ed): IARC, Lyon; 2017. 10:6.

6. Anlauf M, Garbrecht N, Bauersfeld J, et al.: Hereditary neuroendocrine tumors of the gastroenteropancreatic system. Virchows Arch. 2007, 451:S29-38. 10.1007/s00428-007-0450-3

7. Service FJ, McMahon MM, O'Brien PC, Ballard DJ: Functioning insulinoma--incidence, recurrence, and longterm survival of patients: a 60-year study. Mayo Clin Proc. 1991, 66:711-9. 10.1016/s0025-6196(12)62083-7

8. Gagnier JJ, Kienle G, Altman DG, Moher D, Sox H, Riley D: The CARE guidelines: consensus-based clinical 


\section{Cureus}

case reporting guideline development. Glob Adv Health Med. 2013, 2:38-43. 10.7453/gahmj.2013.008

9. Nagy Z, Szabó PM, Grolmusz VK, Perge P, Igaz I, Patócs A, Igaz P: MEN1 and microRNAs: the link between sporadic pituitary, parathyroid and adrenocortical tumors?. Med Hypotheses. 2017, 99:40-4.

10.1016/j.mehy.2016.12.007

10. Zhuang Z, Vortmeyer AO, Pack S, et al.: Somatic mutations of the MEN1 tumor suppressor gene in sporadic gastrinomas and insulinomas. Cancer Res. 1997, 57:4682-6.

11. Capelli P, Martignoni G, Pedica F, Falconi M, Antonello D, Malpeli G, Scarpa A: Endocrine neoplasms of the pancreas: pathologic and genetic features. Arch Pathol Lab Med. 2009, 133:350-64. 10.1043/1543-2165133.3.350

12. Corbo V, Dalai I, Scardoni M, et al.: MEN1 in pancreatic endocrine tumors: analysis of gene and protein status in 169 sporadic neoplasms reveals alterations in the vast majority of cases. Endocr Relat Cancer. 2010, 17:771-83. 10.1677/ERC-10-0028

13. Jiao Y, Shi C, Edil BH, et al.: DAXX/ATRX, MEN1, and mTOR pathway genes are frequently altered in pancreatic neuroendocrine tumors. Science. 2011, 331:1199-203. 10.1126/science.1200609

14. Liu IH, Ford JM, Kunz PL: DNA-repair defects in pancreatic neuroendocrine tumors and potential clinical applications. Cancer Treat Rev. 2016, 44:1-9. 10.1016/j.ctrv.2015.11.006

15. Puccini A, Poorman K, Salem ME, et al.: Comprehensive genomic profiling of gastroenteropancreatic neuroendocrine neoplasms (GEP-NENs). Clin Cancer Res. 2020, 26:5943-51. 10.1158/1078-0432.CCR-201804

16. Mei M, Deng D, Liu TH, et al.: Clinical implications of microsatellite instability and MLH1 gene inactivation in sporadic insulinomas. J Clin Endocrinol Metab. 2009, 94:3448-57. 10.1210/jc.2009-0173

17. Frazier JL, Weingart JD: Metastatic insulinoma to the brain. Endocrinologist. 2009, 19:5. 10.1097/TEN.0b013e31819390e7

18. Yu J, Ping F, Zhang H, et al.: Clinical management of malignant insulinoma: a single institution's experience over three decades. BMC Endocr Disord. 2018, 18:92. 10.1186/s12902-018-0321-8

19. Hoberück S, Michler E, Zöphel K, Platzek I, Kotzerke J, Brogsitter C: Brain metastases of a neuroendocrine tumor visualized by 68Ga-DOTATATE PET/CT. Clin Nucl Med. 2019, 44:50-2.

10.1097/RLU.0000000000002341 